\title{
In the name of the rose: a roadmap for rose research in the genome era
}

\author{
Marinus J. M. Smulders ${ }^{1}$, Paul Arens ${ }^{1}$, Peter M. Bourke ${ }^{1}$, Thomas Debener ${ }^{2}$, Marcus Linde ${ }^{2}$, Jan De Riek ${ }^{3}$, Leen Leus ${ }^{3}$, \\ Tom Ruttink ${ }^{3}$, Sylvie Baudino ${ }^{4}$, Laurence Hibrant Saint-Oyant ${ }^{5}$, Jeremy Clotault ${ }^{5}$ and Fabrice Foucher ${ }^{5}$
}

\begin{abstract}
The recent completion of the rose genome sequence is not the end of a process, but rather a starting point that opens up a whole set of new and exciting activities. Next to a high-quality genome sequence other genomic tools have also become available for rose, including transcriptomics data, a high-density single-nucleotide polymorphism array and software to perform linkage and quantitative trait locus mapping in polyploids. Rose cultivars are highly heterogeneous and diverse. This vast diversity in cultivated roses can be explained through the genetic potential of the genus, introgressions from wild species into commercial tetraploid germplasm and the inimitable efforts of historical breeders. We can now investigate how this diversity can best be exploited and refined in future breeding work, given the rich molecular toolbox now available to the rose breeding community. This paper presents possible lines of research now that rose has entered the genomics era, and attempts to partially answer the question that arises after the completion of any draft genome sequence: 'Now that we have "the" genome, what's next?'. Having access to a genome sequence will allow both (fundamental) scientific and (applied) breeding-orientated questions to be addressed. We outline possible approaches for a number of these questions.
\end{abstract}

\section{Introduction}

Rose is the most well-known and beloved ornamental plant worldwide. As in most other ornamental plant breeding programmes, molecular tools have up to now rarely been used. There is a number of obstacles to implementing molecular breeding in roses. These include its tetraploid nature, the fact that it is vegetatively propagated and that large genetic gains can still be achieved by simple crossing and selection. Moreover, a significant gap exists between research and breeding practices, impeding the application of developed genetic tools in practice. While for large agricultural crops the primary focus is on yield and other quantitative traits for both the academic community and breeders, rose research has

\footnotetext{
Correspondence: Marinus J. M. Smulders (rene.smulders@wur.nl)

${ }^{1}$ Plant Breeding, Wageningen University and Research, P.O. Box 386, 6700 AJ Wageningen, The Netherlands

${ }^{2}$ Faculty of Natural Sciences, Institute for Plant Genetics, Molecular Plant Breeding, Leibniz University of Hannover, Herrenhäuser Strasse 2, 30419 Hannover, Germany

Full list of author information is available at the end of the article.
}

focussed on both characteristics that are qualitative, such as presence/absence of the 'double flower', and some disease resistances, and on complex qualitative traits, including flower colour, scent emission, bud outgrowth ${ }^{1}$, floral development and vernalization response ${ }^{2}$. Although rose is an ideal model species for studying the molecular basis of these traits, they are easily examined by eye by breeders. For instance, scent is a complex trait that is being studied, but it is examined by an experienced nose in a breeding programme.

The worldwide rose research community is relatively small. Therefore, the development of new information and tools for rose breeding and genetics in the past few years has been an important step. The availability of rose genome sequences ${ }^{3-5}$ has made the identification of candidate genes for these traits possible. Once a candidate gene has been found, allelic diversity may be linked to functional diversity (see ref. ${ }^{6}$ ), leading to 'candidate alleles', which is of much use in a highly heterozygous and polyploid crop. Next-generation sequencing has also

\section{(c) The Author(s) 2019}

(c) (i) Open Access This article is licensed under a Creative Commons Attribution 4.0 International License, which permits use, sharing, adaptation, distribution and reproduction in any medium or format, as long as you give appropriate credit to the original author(s) and the source, provide a link to the Creative Commons license, and indicate if changes were made. The images or other third party material in this article are included in the article's Creative Commons license, unless indicated otherwise in a credit line to the material. If material is not included in the article's Creative Commons license and your intended use is not permitted by statutory regulation or exceeds the permitted use, you will need to obtain permission directly from the copyright holder. To view a copy of this license, visit http://creativecommons.org/licenses/by/4.0/. 
facilitated the creation of transcriptomes and large numbers of single-nucleotide polymorphism (SNP) markers, which can be assessed using the 68k WagRhSNP array for rose $^{7}$ or by single SNP assays using flanking sequence information. Owing to newly developed software for dosage scoring and genetic mapping in polyploids, ultradense genetic maps have recently been produced in diploid $^{5}$ and tetraploid rose $\mathrm{e}^{8,9}$ that contain many more markers than maps from previous diploid rose populations (collated in ref. ${ }^{2}$ ). These maps have enabled subsequent quantitative trait locus (QTL) mapping studies ${ }^{10}$ and genome-wide association analyse ${ }^{11,12}$ in tetraploid rose, helping to identify regions of the genome that are statistically correlated with traits of interest. As the genomes and most of the resources are publicly available, rose genomics is far more accessible than ever before.

Here we discuss the opportunities for better understanding of structural variation in the rose genome and genome evolution in the genus Rosa and applications for QTL mapping, genome-wide association study (GWAS) analysis and functional analyses of traits, and for measuring genetic diversity, which, we hope, will ultimately improve the speed and precision of breeding new rose cultivars.

\section{Genome sequence and genomic tools in rose}

\section{The rose genome sequences}

Nakamura et al. $^{3}$ released the first rose genome sequence from the wild and heterozygous Rosa multiflora. It was still a genome in pieces (low N50 and 83,189 scaffolds, Table 1). The recent release of two high-quality reference genomes, obtained by sequencing haploids using a combination of short and long reads, represents a tremendous improvement with $\mathrm{N} 50$ of 3.4 and $24 \mathrm{Mb}$ and 551 and 82 scaffolds, respectively (Table 1). Pseudomolecules, corresponding to the chromosomes, were obtained by anchoring the new sequences to a highdensity diploid SNP genetic map ${ }^{5}$ or to the high-density map for tetraploid rose of Bourke et al. ${ }^{8}$, and validated by $\mathrm{HiC}$ sequencing ${ }^{4}$. Details concerning genes and transposable element annotation are listed in Table 1. The genomes are publicly available at $\mathrm{GDR}^{13}$, NCBI and some dedicated websites (Table 1).

A comparison of the two independent assemblies of the haploids of Rosa chinensis 'Old Blush' indicates that at they are essentially co-linear, indicating a high level of accuracy at both the contig and scaffolding level. Some assembly discrepancies appear as rearrangements (Fig. 1). These rearrangements may be due to assembly problems or they may highlight structural differences between the two haplotypes in 'Old Blush', which has been described as an interspecific hybrid ${ }^{14}$. One local rearrangement that has been studied in detail is at the continuous-flowering locus, where a large inversion led to the loss of the RoKSN gene (Fig. 1, ref. ${ }^{5}$ ). The R. multiflora genome $\mathrm{e}^{3}$ has not been compared to the other two as it is much more fragmented.

\section{Transcriptomics resources}

Next-generation sequencing has also facilitated the creation of transcriptome data of various tissues and developmental stages for several genotypes and species (e.g. see refs. $\left.{ }^{15-18}\right)$. Such data can be further used to perform gene curation and annotation. As more and more transcriptomic data will be available with their associated metadata, a gene expression atlas can be developed; such tools will help to select for candidate genes and perform meta-analysis.

\section{Molecular markers}

SNPs are abundantly present as genetic variation between and within accessions, their detection can be automated and they are easily linked to genome sequences (based on the flanking regions). Therefore, they are currently the marker of choice. Large numbers of SNP markers have been generated in rose using genomic or

Table 1 Principal metrics of the recent published rose genome sequences

\begin{tabular}{|c|c|c|c|}
\hline Genotype & Haploid of 'Old Blush' & Haploid of 'Old Blush' & Rosa multiflora Thunb. \\
\hline Methods & Illumina/PacBio & Illumina/PacBio & Illumina \\
\hline N50 & $3.4 \mathrm{Mb}$ & $24 \mathrm{Mb}$ & $90.8 \mathrm{~kb}$ \\
\hline No. of contigs & 551 & 82 & 83189 \\
\hline Total genome size & $512 \mathrm{Mb}$ & $515 \mathrm{Mb}$ & $740 \mathrm{Mb}$ \\
\hline \multicolumn{4}{|l|}{ Annotated genes } \\
\hline Coding & 39,669 & 36,377 & 67,380 \\
\hline Non-coding & 4812 & 3971 & ND \\
\hline Transposable elements & 61.8 & 65.2 & 56.4 \\
\hline Class I & 35.1 & 31.6 & 12.3 \\
\hline Class II & 11.7 & 11.6 & 2.4 \\
\hline Other & 15 & 22 & 39.2 \\
\hline Reference & 5 & 4 & 3 \\
\hline Dedicated websites & https://iris.angers.inra.fr/obh/ & https://lipm-browsers.toulouse.inra.fr/pub/RchiOBHm-V2/ & http://rosa.kazusa.or.jp/ \\
\hline
\end{tabular}

N50 represents the length of the contig in order to have $50 \%$ of the total assembly contained in contigs larger than this value. ND for non determined 


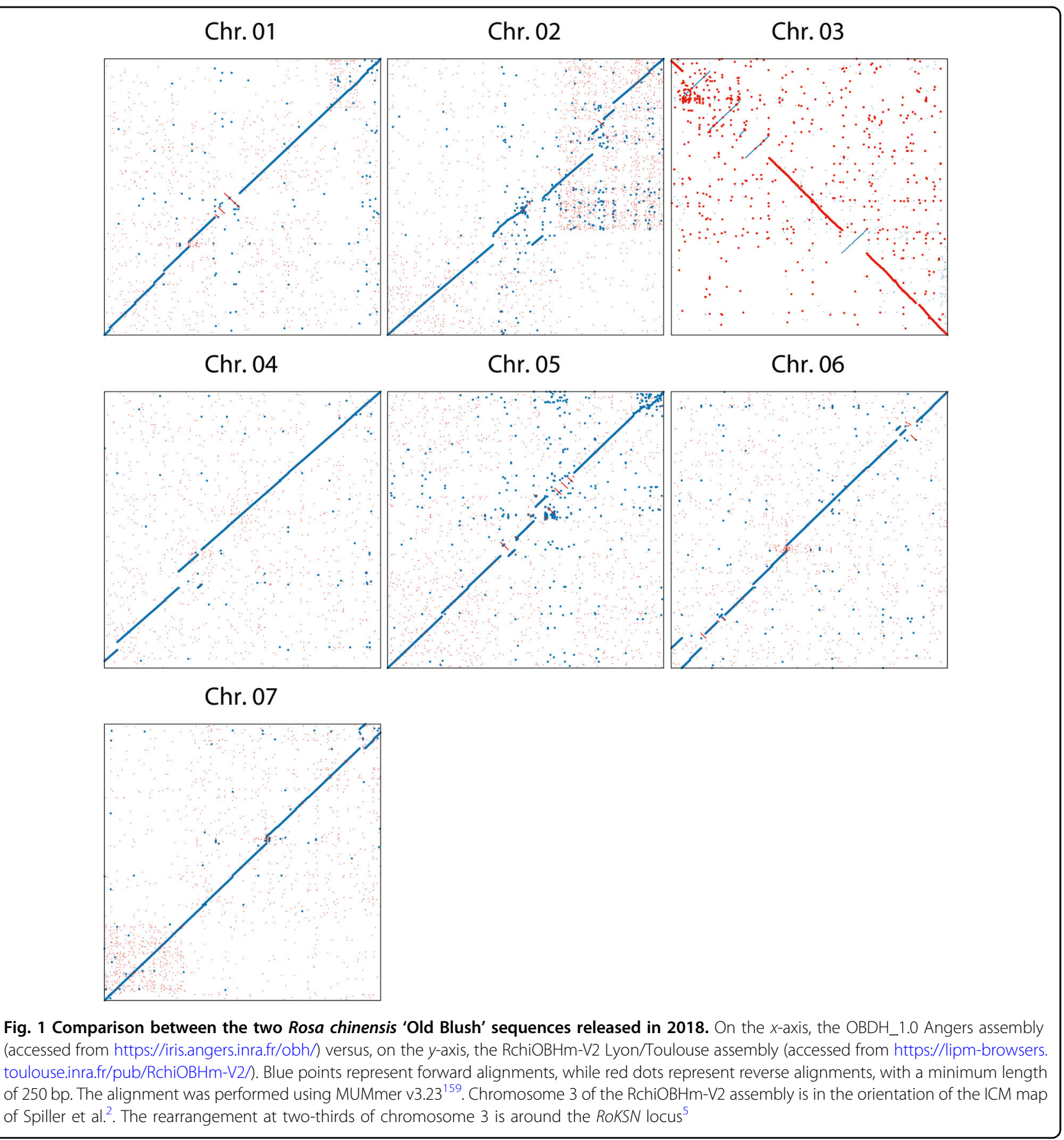

transcriptomic sequences ${ }^{19,20}$. The $68 \mathrm{k}$ WagRhSNP Axiom SNP array ${ }^{7}$ was designed to genotype more than 68,000 SNPs identified from transcriptome sequences from 12 modern garden roses, two cut rose cultivars and a R. multiflora hybrid ${ }^{7}$. PCR-based marker systems, for example, KASP (Kompetitiv Allele-Specific PCR) can be used for single SNP marker genotyping as well. Since many SNP markers are polymorphic in both garden and cut roses, and in diploid as well as tetraploid roses, there are now many publicly available resources to identify regions in the genome that are associated with traits of interest.

Current and future comparisons across studies will benefit if this SNP array, or a subset of the SNPs on the array, is used for genotyping across different studies. For instance, it would become possible to expand the sample size of a GWAS study, or to do a combined re-analysis on data from independent GWAS panels.

Biallelic SNPs can provide similar levels of discerning power as multi-allelic simple sequence repeat (SSR) 
markers if a sufficiently large number of SNP markers is employed. The power to distinguish multiple alleles at a locus may also be increased by combining a number of neighbouring SNP markers into a single multi-allelic marker and analysing them as haplotypes ${ }^{21}$. Haplotypes also carry information on identity by descent. The first strategies have been developed to infer phasing of haplotypes from genotype data; however, they have a low efficiency when ploidy increases ${ }^{22}$. Sequencing technologies such as Pacific Biosciences and Oxford Nanopore produce longer sequencing reads, and therefore also longer (and potentially more informative) haplotypes.

Re-sequencing for diversity analysis avoids possible ascertainment bias while offering allele dosage estimates from read proportions ${ }^{23-25}$, although various steps in the actual protocol used may negatively affect the reliability of dosage estimation in polyploids. Re-sequencing at very low coverage per individual (skim sequencing) or the use of a complexity reduction $\operatorname{step}^{26}$, such as restriction-siteassociated DNA sequencing or genotyping by sequencing (GBS), has been proposed to reduce cost, and indeed have already been applied in $\operatorname{rose}^{5,27,28}$. Population genetics analysis that do not require individual genotype information (Pool-seq) reduces the number of libraries, while protocols for reducing the costs of libraries have also been developed ${ }^{29}$.

As most cultivated roses are complex hybrids, Insertion/ Deletion variants (InDels) occur frequently ${ }^{5}$. Indels thus represent an interesting source of genetic variation. Resequencing will include them; however, indels are difficult to detect in a reliable way as variant calling programmes often disagree on the detection of indels. Hence, the quality (reproducibility) is often much lower than for SNP calls. In Citrus it was shown that indels can help account for the contribution of ancestral species ${ }^{30}$.

\section{Genotyping and genetic mapping}

SSR (or 'microsatellite') markers have been widely employed in genetic diversity and mapping studies in rose. Several genetic maps have been produced with SSR markers and other markers, including amplified fragment length polymorphism (AFLP) and nucleotide binding site (NBS) to map traits, mostly in diploid crosses. These maps have been integrated by Spiller et al. $^{2}$ into a consensus map. The numbering and orientation of this map, with annotated traits, have subsequently been used for highdensity SNP array-based maps, which in turn were used for anchoring and ordering the rose genome sequences, thus tying together most genetic mapping and QTL studies in rose. As the SNP markers were derived from expressed sequences, this gave a focus on the part of the genome where most genes reside and where most recombinations occur during meiosis, and thus to a high- quality haploid genome assembly in those regions that are of the highest interest.

For polyploids, SNP array data currently provided the clearest information on the allele dose of markers. Using the dosage of SNP markers, tetraploid SNP genotypes can be used for linkage analysis with dedicated polyploid mapping software such as TetraploidSNPMap ${ }^{31}$ or polyMapR $^{10,32}$. Ultra-dense genetic maps thus have recently been produced in diploid ${ }^{5}$ and tetraploid rose ${ }^{8,9,33}$, either for separate homologous chromosomes, or integrated across chromosomal linkage groups (LGs). Yan et al. ${ }^{27}$ made a map using SNPs in GBS data. The dense SNP maps have enabled subsequent QTL mapping studies ${ }^{34}$ and genome-wide association analyses ${ }^{11,12}$ in tetraploid rose, helping to identify regions of the genome that are statistically correlated with traits of interest.

\section{Genetic diversity in rose}

Historical rose breeding involved a complex sequence of interspecific hybridizations between seven to fifteen wild species that contributed to the germplasm of modern roses (the number depends on the interpretation and importance attributed to their contribution to the domestication), thus shaping the genomes of modern cultivars after three centuries of breeding ${ }^{35,36}$. This atypical genetic bottleneck means that genetic diversity in roses now encompasses two sources of sequence variation. On the one hand, genetic diversity in the genus Rosa comprises 140 to 180 wild species, including all genetic variation therein; on the other hand, it comprises a tremendous number of rose cultivars, currently estimated to exceed 30,000 cultivated cultivars ${ }^{37}$.

Several studies on genetic diversity in rose have been performed with SSR markers ${ }^{38-42}$, mostly in the cultivated germplasm. Phylogenetic studies have been conducted on Rosa species across the genus, and on modern rose cultivars, indicating close relationships between different botanical sections and horticultural groups (refs. ${ }^{42-48}$ ). These studies have shed some light on general patterns of genetic diversity, but give little information on the contribution of specific parents in hybridizations to desired traits.

\section{Rose domestication}

Two important rose species can be considered to be domesticated in the common sense of the term: the tetraploid Rosa gallica in Europe ${ }^{49}$ and the diploid R. chinensis in Asia ${ }^{39}$. The most remarkable recent insights in the origin of modern cultivated roses concern the study of the genetic structure of old Chinese garden roses ${ }^{39}$, their large introgression in the European germplasm since the eighteenth century ${ }^{42}$ and the subsequent selection during the twentieth century within a subsample of these hybrid garden roses to obtain cut roses ${ }^{40}$. In contrast, almost nothing is known on the origin of the roses that are used 
for the production of essential oils: Rosa damascena and Rosa centifolia.

A comparative analysis of these two domestication events in different parts of the world has not been carried out yet. It would involve wild and early cultivated genotypes of the two species and may reveal the precise location of domestication events by comparison to wild gene pools, the presence of potential genetic bottleneck(s) during the domestication event and even the timing of domestication events. Generally, the domestication of perennial species, often with long intergenerational time, is more recent than most annual crops and involves shorter or even no genetic bottlenecks, leading to a reduced domestication syndrome. Rose, as a perennial species with a short juvenile phase, may have an intermediate pattern. Next to testing the impact of ploidy level on domestication dynamics, the comparison of regions targeted by selection in $R$. gallica and $R$. chinensis and the domestication syndrome traits in common between the two species (double flower, colour diversification) or specific to one of the species (recurrent blooming in Rosa chinensis, abiotic stress tolerance in Rosa gallica) could clarify the phenomenon of convergent or differential domestications. As in other perennials, including apple, citrus and olive $e^{50-52}$, introgressions may have played an important role in the domestication of rose.

\section{Mosaic genomes in modern roses}

Species hybridizations may have led to 'mosaic' genomes, which are formed by small parts of discontinuous ancestral genomes, as has been observed in cultivated Citrus $^{50}$. Re-sequencing of the first modern rose cultivar 'La France' and three or four species each from the sections Cinnamomeae, Synstylae and Chinenses showed that large regions of chromosome 2, 3 and 5 of the triploid cultivar 'La France' have a strictly Chinenses origin, while other regions of chromosome 2, 5 and 7 had a Synstylae origin only ${ }^{4}$. This study on 'sequence signatures' is the first step towards a more comprehensive understanding of the history of the rose breeding process.

Owing to the vegetative propagation of cultivars since their commercial introduction, the original genome constitution can still be accessed and sequenced and thus 300 years of rose breeding history can be reconstructed. Sequencing of founder individuals of subsequently developed horticultural groups, or cultivars with great commercial or breeding success, may reveal the origin of ancestral genome segments (sequence signatures). Reconstructing the segregation of these 'founder haplotypes' throughout centuries of breeding as captured in cultivar collections, while correlating these genomic segments with the evolution of horticultural traits in roses, may identify signatures of selection. In addition to 'La France', a broader study of various Tea hybrids may show if the observed patterns of single ancestry of specific chromosomic regions were targeted by breeders or if they appeared by chance, by researching over-representation of a given ancestry in the pedigree. This approach has already been used in apple, peach, cherry and strawberry to identify haplotypes passed on by highly successful parents during the breeding process ${ }^{53-59}$. Identification of genealogical relationships between cultivars ${ }^{60}$ can be used for validation.

Understanding the mosaic genomes of modern cultivated roses may also detect the occurrence and determine the impact of structural divergence between hom(e)ologous chromosomes during meiosis. A study of pairing behaviour between tetraploid rose parents of a segregating population showed evidence for 'segmental allotetraploidy': pairing of some chromosomes was preferential (partially disomic) while most other chromosomes behaved tetrasomically ${ }^{8}$. Sometimes, these two modes of inheritance were observed in segments of the same chromosome, in the meiosis of one of the parents. A comparison of chromosome pairing behaviour during rose meiosis and the occurrence of structural variation between hom(e)ologous chromosomes across several meiosis in different genetic backgrounds is needed in order to understand the link between these two phenomena, including which type(s) of structural variation exist. Alternatively, the behaviour may be genetically governed. In the latter case the mechanism may not be rose-specific. Nevertheless, rose is a suitable model crop for such studies as several species and much historical material (old cultivars produced involving species crosses) is available. A broad analysis of the modes of inheritance in various modern rose cultivars, but also in old garden roses and wild roses, should clarify whether 'segmental allotetraploidy' occurs commonly in the genus Rosa or whether it is genotype-specific, and whether it occurs more frequently in some subsets of the rose germplasm than others.

\section{Sports}

Rose cultivars are clonally multiplied through vegetative propagation. However, mutations occur frequently, leading to variation, sometimes visible phenotypically ('sports'). Most of this variation is thought to be due to point mutations, although transposon activity and epigenetic effects cannot be ruled out. Consistent with point mutations, original cut rose varieties and mutants thereof were identical with 11 SSR markers, so mutant families could easily be detected, while all seedling-derived varieties could be clearly distinguished from them and from each other (similarity $<0.90)^{61}$.

Comparing the genome of pairs of sport and original cultivar may provide information on genomic regions that explain mutant phenotypes and help to understand why 
some traits are more often involved in sports, such as climbing growth habit or petal colour changes.

\section{The Canina meiosis}

Species of the Caninae section display a unique type of asymmetric meiosis that has not been described in other angiosperm species ${ }^{62,63}$. It also appears in Alba roses, a European Old garden rose group, which could be hybrids between sections Caninae and Gallicanae ${ }^{64}$. The Caninae section is composed of tetraploid, pentaploid and hexaploid species. Irrespective of the ploidy level, the Caninae asymmetric meiosis involves the formation of one set of bivalent-pairing chromosomes and the remaining chromosomes are univalent (unpaired). One reduced set of bivalent chromosomes and all the univalent chromosomes are included in an egg cell, while the other reduced bivalent chromosome set is transmitted by pollen cells. It was shown by molecular markers and fluorescent in situ hybridization that the two bivalent-forming chromosomes are highly homologous, whereas univalent chromosomes are more divergent ${ }^{65,66}$. Genome sequencing of several species of the section Caninae may help to reveal whether structural variation among bivalent and univalent chromosomes is the primary cause of asymmetric meiosis or if and how the decision per chromosome to pair or not to pair is genetically controlled. Inheritance of haplotypes or complete chromosomes through pollen and egg cells can be most efficiently studied using a dense array of SNP markers. The software package polymapR can deal with odd ploidies and with offspring of plants with different ploidy level ${ }^{10,32}$. Based on the offspring data preferential chromosomal pairing in each parent can be detected, and all meiotic recombination events would become visible.

\section{Structural genomic variation in rose Interspecific genomic variation}

In the genus Rosa the 2C DNA amount in diploid species ranges from $0.78 \mathrm{pg}$ in Rosa xanthina (section Pimpinellifoliae) to $1.29 \mathrm{pg}$ in 'Félicité and Perpétue' (hybrid Sempervirens) ${ }^{67}$ and from $0.73 \mathrm{pg}$ in $R$. zhongdianensis (section Pimpinellifoliae) to $1.77 \mathrm{pg}$ in $R$. brunonii (section Synstylae) ${ }^{68}$. The recently sequenced $R$. chinensis is among the largest genomes known among diploid roses (estimated at $1.16 \mathrm{pg}^{67}$ to $1.67 \mathrm{pg}^{68}$ ). Roses from the Pimpinellifoliae section generally have the smallest genome size, while Synstylae roses have the largest genomes ${ }^{68}$.

Genome size variation in angiosperms is typically associated with two types of events: whole-genome duplication (WGD) or transposable element amplification $^{69,70}$. With regard to the latter, approximately $68 \%$ of the $R$. chinensis reference genome sequence consists of transposable elements, especially long-terminal repeat retrotransposons like Gypsy and Copia elements ${ }^{5}$. For most transposable element families, a two-fold higher abundance was found in Rosa as compared to Fragaria $v e s c a^{5}$, explaining a substantial part of the genome size difference between $R$. chinensis and $F$. vesca. Shallow shotgun sequencing of a comprehensive sets of species across the genus Rosa and subsequent clustering and quantification of repetitive sequences will reveal if species-specific repetitive elements exist. More extensive (re)sequencing will be needed to ascertain whether differential amplification of transposable elements can explain the variation in genome size among rose species.

Besides the role of transposable elements in genome size evolution, the insertion of copia elements into specific protein-coding genes ${ }^{5,71}$ gave rise to two of the most important horticultural traits: double flower and recurrent blooming (see below). Rose breeding in general may have favoured species from sections with large genomes, as transposon activity is known to create allelic diversity ${ }^{72}$. A related question is whether retrotransposon activity is higher in tetraploid taxa compared to diploids, and whether this has also led to functional variation that is useful for the ornamental value of roses.

Whole-genome assembly and gene prediction has revealed no signs of recent WGD events in $R$. chinensis $^{4,5}$. In addition, despite the difference in genome size, the $240 \mathrm{Mb} F$. vesca genome contains 34,809 predicted genes $^{73}$, while the $560 \mathrm{Mb}$ 'Old Blush' R. chinensis genome has only fractionally more predicted genes $\left(39,669\right.$ genes ${ }^{5}$; 36,377 genes $\left.^{4}\right)$.

\section{Rose comparative genomics}

From an evolutionary point of view, rose is a very interesting model species as it includes species at several ploidy levels as well as many cultivars with a hybrid ori$\operatorname{gin}^{74}$. The sequencing of thousands of individuals in Arabidospis thaliana ${ }^{75}$, rice $^{76}$ and maize $\mathrm{e}^{77}$ has demonstrated extensive differences in genome constitution even among accessions within a species, leading to the definition of 'core' genes (which are present in all members of a species), and 'distributed' or 'dispensable' genes (present in a subset of members). The 'pan-genome' represents the full genome complement across all sampled members.

Metagenome-like assembly strategies in rice ${ }^{76}$ and an analysis of 3010 re-sequenced rice accessions ${ }^{78}$ revealed that 'distributed' gene families showed enrichment in regulation of immune and defence responses. Other studies also unveiled their role in adaptation to abiotic and biotic stresses ${ }^{79}$, species diversification and development of novel gene functions ${ }^{80}$. (Meta)genome assembly and gene annotation of species across the Rosa genus and in closely related species, and subsequent comparative genomics will be required to define whether rose also has conserved and lineage-, section- and/or species-specific genes. One of the possible hypotheses is that resistance 
$(R)$ genes behave as a dispensable group gene family, while susceptibility $(S)$ genes generally would be members of core gene families. For such questions to be answered, it will be necessary to collect and analyse genome sequences of species and accessions across the genus that represent the taxonomic diversity, but also the diversity in various abiotic and biotic conditions in which roses grow.

Among the most interesting cases to study in roses is the question of resistance genes. The sequencing of bacterial artificial chromosome (BAC) clones around the $R d r 1$ locus, contributing to the rose black spot resistance, in $R$. multiflora (9 TIR-NBS-LRR (TNL) genes) and $R$. rugosa (11 TNL genes) enabled both rearrangements and duplications in this locus to be discovered ${ }^{81,82}$. A larger analysis of clusters of resistance genes in roses is needed to understand how their organization and evolution can be associated with resistance levels.

\section{Important traits in rose}

For decades, genetic and physiological approaches have been developed to decipher ornamental traits and to identify regions of important genes controlling these traits $^{83}$. We will briefly summarize the most important findings and the main issues for current or future research in these traits since the publication of the rose genome sequence. We will focus amongst others on floral traits, including the mode and date of blooming (once-flowering versus continuous flowering), simple versus double flowers, petal development and flower colour. Besides an interest from developmental biology in the mechanisms, these traits are also important targets for breeders (see section 'Rose breeding').

\section{Mode of blooming: recurrent versus once-flowering}

Wild roses flower in spring over a few weeks. Those that have the ability to bloom again after the first blooming are described as recurrent blooming. There are many recurrent blooming species in the ornamental sector, including Jasmine, Carnation, Hydrangea, Pelargonium and Lavender. After the first flowering period, a recurrent blooming rose has the ability to flower a second time, either continuously during the favourable season (continuous flowering habit) or later in the season, which may be occasional (occasional re-blooming habit) ${ }^{71}$. The continuous-flowering phenotype is caused by a mutation in a TFL1-like flowering repressor gene (RoKSN). Two alleles leading to recurrent flowering have been described: one is due to the insertion of a copia transposable element $^{71}$ and the other is due to a large rearrangement at the RoKSN locus, leading to the deletion of the gene ${ }^{5}$. In both cases, the floral repressor is not produced and the roses flower continuously. In once-flowering roses, the expression of $R o K S N$ is repressed in spring when the plant flowers and this is regulated by GA ${ }^{84}$. The RoKSN protein forms a complex with RoFD and competes with RoFT, a floral activator for repression of flowering ${ }^{85}$.

Presently, only extreme phenotypes have been studied (continuous versus once-flowering). Sequencing of alleles of the genes involved in flower initiation and flowering time in several cultivars and species by either wholegenome sequencing or targeted re-sequencing may improve the understanding of the molecular bases of intermediate phenotypes (equilibrium between vegetative and floral development). This may lead to the characterization of new RoKSN alleles or new loci controlling recurrent flowering or responding to environmental cues; for instance, by relating the genetic variation of RoKSN alleles to precise phenotyping of flowering (counting the number of flowers in time and space) as initiated on the TFL1/FT family genes by Wang et al. ${ }^{86}$. Association mapping across germplasm with a wide range of phenotypic differences in flowering may detect new loci. These genetic approaches can be extended by a functional analysis, such as knocking down RoKSN expression in onceflowering roses, as was previously demonstrated in Fragaria.

\section{Number of petals}

Flowers of wild roses have five petals. During domestication and selection of roses, flowers with numerous petals, called 'double flower', have been selected. Up to 517 petals have been observed in a single flower ${ }^{87}$. It has been proposed that the double flower phenotype is due to dysregulation of the $A B C E$ genes during floral development ${ }^{88}$. A dominant gene located on $\mathrm{LG}^{2,89}$ controls single versus double petals (with the double flower phenotype being a dominant qualitative trait). Using a GWAS panel, it was proposed that this locus also controls the number of petals of the double flowers ${ }^{5}$, next to two QTLs on LG2 and LG5 ${ }^{87}$. Hibrand Saint-Oyant et al. ${ }^{5}$ identified a rose APETALA2/TOE homologue within the QTL region on LG3 as most likely candidate gene for the major regulator of petal number in rose. Silencing it decreased the number of petals ${ }^{90}$. It was proposed that the insertion of a TE in the eight intron is responsible for transcription of a messenger RNA without a miR172 binding site, leading to a deregulation of the APETALA2/TOE homologue $\mathrm{e}^{91,92}$.

The ideal number of petals for cut roses, from a commercial point of view, is between 40 and 60 petals, while a greater range occurs in commercial garden rose cultivars. Understanding the interaction between the major gene and other QTLs, in relation to environmental signals, will help to develop a model for petal number development and the effects of different combinations of alleles to obtain the desired inflorescence. For this we need to determine allelic diversity at this and other loci, look at 
expression differences, and experimentally test the effects of different combinations of alleles.

\section{Petal colour and development}

Flower colour plays an important role in the attractiveness of rose flower for insects, but also for humans; colour therefore has an important aesthetic value. Most flower colours are present in the genus Rosa, except for blue, and a large diversity in the colour variants is available, including colour intensity and the organization of colour on the petals. These colours come from different genetic backgrounds: pink and purple from Rosa rugosa, and yellow from Rosa ecae, Rosa foetida and Rosa hemisphaerica. The orange colour, induced by the pelargonidin pigment, was introduced by Kordes. The darkercoloured central zone of rose flowers was probably derived from Rosa persica.

Yellow and orange colours are the result of carotenoid pigment accumulation, while red colours are explained by anthocyanin accumulation, mainly glycosylated xanthocyanidin $^{93,94}$. In rose, a unique glycosyltransferase enzyme performs glycosylation at two different positions ${ }^{95}$. Different homologues of anthocyanin biosynthesis pathway genes are accumulated during pigment accumulation in the rose petals of 'Old Blush' ${ }^{\text {,93 }}$. A blue/violet colour is usually the result of accumulation of delphinidin-based anthocyanins. The absence of delphinidin in rose is attributed to the lack of flavonoid $3^{\prime}, 5^{\prime}$-hydroxylase ${ }^{96}$. Recently, a connection between colour and scent emission was proposed through the action of SPL9-miRNA156, putatively controlling the synthesis of anthocyaninin and Germacren D ${ }^{4}$.

The genetic inheritance of flower colour was studied in different genetic backgrounds and a number of QTLs have already been identified ${ }^{2,97}$. Owing to a detailed annotation of carotenoid-related and flavonoid biosynthetic genes in the rose genome ${ }^{3,4}$, co-localization between genes and QTLs can now be investigated and candidate genes analysed. This may help to answer some intriguing questions regarding colour in rose: (i) Why is the red colour predominant in modern roses, (ii) why it is difficult to obtain a bright yellow colour and (iii) what are the origins of variegation?

\section{Self-incompatibility}

In rose, self-incompatibility (SI) ${ }^{98}$ was proposed to be gametophytic as in other Rosaceae, where the S-locus is composed of genes coding for F-box and S-RNase proteins, as the male and female components of SI, respectively ${ }^{99}$. In rose, the $\mathrm{S}$-locus was mapped on $\mathrm{LG} 3^{2,100}$, in a region where S-RNase and F-box genes were located in the reference genome ${ }^{5}$. Transcripts of one S-RNase and one F-box gene accumulate in pistils and stamens, respectively. Furthermore, the $\mathrm{OB}$ genome sequence allowed the analysis of a region so far only covered by molecular markers linked to the SI phenotype. This region is syntenic with the S-locus in Prunus persica ${ }^{5}$.

\section{Fragrance}

Fragrances in garden roses are very diverse and scent has always been an important trait in the selection process. However, not all marketed roses are heavily scented, despite the effort of breeders. In particular, roses bred for the cut flower market often lack scent. In modern roses or Hybrid Tea roses, scent is mainly produced by the petals, although stamens can also contribute ${ }^{101}$. Hundreds of volatile molecules, belonging to different biosynthetic pathways, have been isolated from rose petals. The combination of these molecules generates the particular rose scent bouquet. The biosynthetic pathways of many rose scent compounds are not completely known. The biosynthesis of 3,5-dimethoxytoluene, responsible for the 'tea scent' of some cultivars, involving $O$-methyltransferases, was the first to be deciphered ${ }^{102,103}$. The pathway leading to 2-phenylethanol (2PE) was also studied in detail, with the identification of the key enzymes phenylacetaldehyde synthase $^{104}$ and phenylacetaldehyde reductase ${ }^{105}$. Recently, an alternative pathway, which is seasonally induced in summer, has been identified in roses for the production of 2PE. This new pathway uses aromatic amino acid aminotransferase ${ }^{106}$ and phenylpyruvate decarboxylase ${ }^{107}$.

Terpenoids, especially monoterpene alcohols such as geraniol, are also major constituents of rose flower volatiles, mostly responsible for the 'typical rose scent'. Generally, terpenoid biosynthesis in plants is achieved by various terpene synthases ${ }^{108}$. However, with a combination of transcriptomic and genetic approaches, it was recently discovered that rose uses a terpene synthaseindependent pathway. A key enzyme of this pathway is RhNUDX1, belonging to the Nudix protein family. A positive correlation was found between the expression levels of RhNUDX1 and the production of the monoterpenoid geraniol, indicating the essential role of this protein in scent production in roses ${ }^{109}$. Despite these biochemical studies, knowledge on fragrance biosynthesis is still incomplete and cannot yet be used to assist breeders in scented rose selection. Indeed, very few genetic approaches, including QTL and GWAS, have been used to analyse scent genetic determinism in rose. The first published analysis of scent was performed using a segregating tetraploid rose population ${ }^{110}$. This analysis showed that a large proportion of offspring lacked fragrant volatile compounds, so that scent may have been lost by such a cross. Another study of genetic determinism of rose compounds was conducted in diploid roses ${ }^{2}$. Several QTLs influencing volatile contents were found, but no 
functional relationships to known candidate genes were demonstrated in this study.

Recently, Roccia et al. ${ }^{6}$ identified a QTL that colocalized with a gene involved in the pathway for 2PE, $R h P A A S$, the expression of which was responsible for the capacity of descendants to produce $2 \mathrm{PE}$. The identification of genes underlying all QTLs and their functional characterization would be of great interest to elucidate the control of aroma volatile levels in rose petals. Next to QTL studies, association studies could be applied on scent traits. Once the key genes involved in the biosynthetic pathway of a compound are known, the major challenge is to understand their evolution in the genus Rosa and how favourable or unfavourable alleles were selected during evolution and domestication. For that purpose, more transcriptomic datasets of roses with contrasting scent profiles are needed, and re-sequencing data. Genetic analysis of diploid or tetraploid populations, in which the alleles from a strong-scented parent segregate, would enhance the power of such approaches.

\section{Vase life}

Concerning petal development and senescence, which is an important trait for the vase life of cut flowers, Ma et al. ${ }^{111}$ reviewed the most important gene networks and the implication of different hormones in ethylenesensitive flowers, among which rose features. Putative key regulating genes can now be targeted to study the effects on vase life. One way to do that may be to generate an inventory of the allelic diversity of the key regulating genes across the germplasm, followed by crossing and functional studies. As senescence may also cause susceptibility to fungal diseases such as botrytis, this type of research could also offer important links to disease resilience in garden roses.

\section{Disease resistance}

The most important infectious diseases of roses are: downy mildew (Peronospora sparsa), powdery mildew (Podosphaera pannosa), black spot (Diplocarpon rosae), spot anthracnose (Sphaceloma rosarum), crown gall (Agrobacterium tumefaciens) and the rose rosette virus. Among the insect pests, rose aphids and thrips are critical for production, in particular for the production of cut and pot roses. To date, only few commercial rose cultivars have significant levels of disease resistance ${ }^{112}$, although it has become more important in the breeding process, especially for garden roses. The analyses of the inheritance of resistance to diseases revealed either single genes (e.g. against black spot $R d r 1-4^{33,81,113,114}$, powdery mildew $\left(\mathrm{mlo}^{115}\right)$ or QTLs (e.g. against powdery mildew $\left.^{116,117}\right)$.

Based on the complete rose genome, resistance loci can now be linked to groups of candidate genes. This is facilitated by the fact that most $R$ genes belong to the large group of NBS-LRR genes. Further, a complete genome enables markers to be generated from the complete set of putative $R$ genes, as demonstrated by the RenSeq technique using capture-based sequencing ${ }^{118}$. This will speed up the localization of $R$ genes $^{119}$, also in wild rose species not yet used in breeding.

In cases where candidate $R$ genes cannot be immediately identified, the genome assembly greatly facilitates the generation of additional, more closely linked SNP markers in those $R$-gene regions currently only loosely covered by molecular markers. This will be of importance for recently mapped loci, for example, $R d r 3$ and $R d r 4$, where the most closely linked markers define a region of approximately $9 \mathrm{cM}$ in which no further recombinations have been identified ${ }^{32,114}$.

\section{Plant architecture}

Plant architecture relates to vigour, productivity and density (which may influence the incidence of diseases). In addition, new forms and shapes, including compact plants (for use in urban areas), may be needed to maintain the current rose market share or open up new markets. Progress in research in this area has been slow. Studies on rose plant architecture have been done using manual phenotyping of architectural components ${ }^{120-122}$ or with three-dimensional digitalization ${ }^{123}$. These analyses require fully developed plants and are time consuming. Nevertheless, they have produced a number of QTLs, and markers have been identified for these QTLs, showing the complexities of these characters. Scientific progress will depend on our ability to identify functional genes from relevant developmental pathways in these QTL regions, on whether allelic effects remain as strong when transferred into other genetic backgrounds and their interaction with environment and cultural practices. High-throughput phenotyping systems may not only make it less laborious to study plant development, but they may also pinpoint certain short stages in development in which genetic differences manifest themselves more clearly.

\section{Speeding up functional gene identification}

Previously gene cloning was a long and laborious process. One approach involved positional cloning and looking for co-localization with candidate genes, as was done for the continuous-flowering gene of rose ${ }^{71}$. Alternatively, map-based cloning and BAC library sequencing was used, as was done for $R d r 1$, the first resistance gene for black spot ${ }^{81,124}$. The reference genome of $\operatorname{rose}^{3-5}$ opens new opportunities to rapidly isolate and characterize important loci by combining genetic, genomic and transcriptomic approaches. Here, we review various schemes that may accelerate the identification of 
a.

LG 3

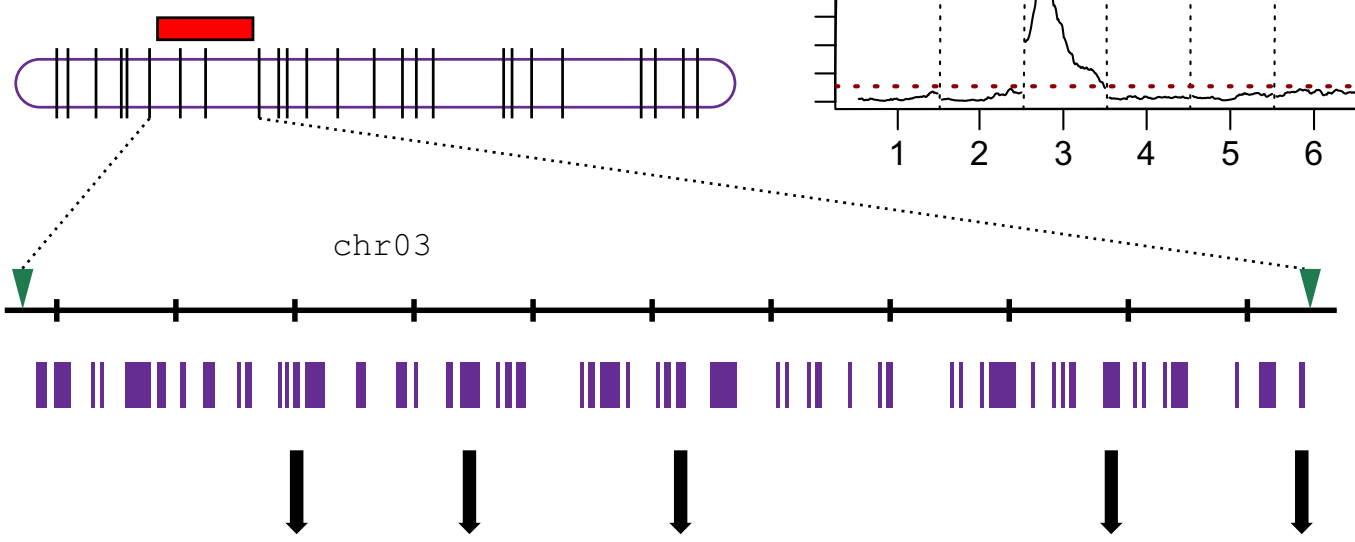

b.
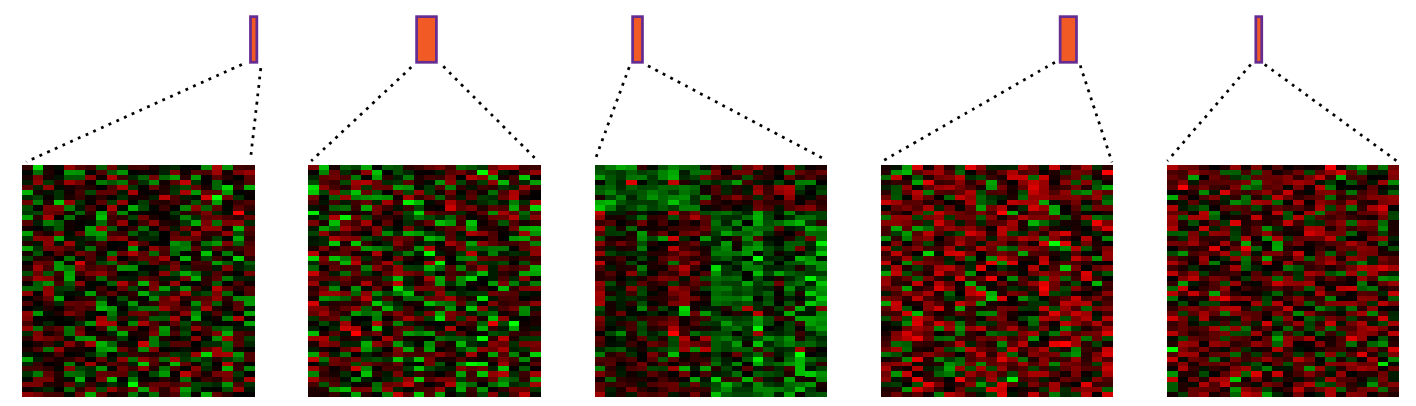

d.

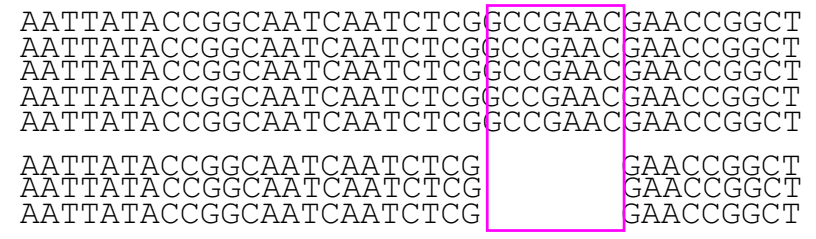

Fig. 2 Illustrative scheme for gene cloning by combining genetic, genomic and transcriptomic approaches. a Using genetic approaches (such as quantitative trait loci (QTL) mapping in F1 progenies or association analysis across a panel of accessions), major genes or QTLs can be detected for important ornamental traits and located on the seven rose linkage groups. $\mathbf{b}$ In the corresponding region of the rose genome sequence, using the functional annotation of the rose genome (each purple box represent an annotated gene), putative candidate genes can be identified based on similarities with genes known to be involved in the studied process in model plants such as Arabidospis thaliana. In the candidate genomic region, five genes have been identified with possible roles (pink boxes), as transcription factors. c By transcriptomic approach, a differentially expressed gene between two contrasting conditions for the studied trait can be identified. $\mathbf{d}$ For the gene with contrasting expression, allelic variants can be identified by sequencing mutant pairs or diversity panels. In this example, two alleles are detected, which differ by an indel. Other variants can be single-nucleotide polymorphisms (SNPs) (synonymous or non-synonymous) or insertions of transposable elements

functional genes and validation of the functional allele thereof in rose owing to the availability of reference genomes.

A schematic, idealized work-flow is shown in Fig. 2. A major gene, located on LG 3 of the genetic map, is identified by QTL mapping or a GWAS analysis (Fig. 2a). Flanking markers delimit the corresponding region in the genome sequence, in which five genes have been annotated that may be good candidates for the trait
(Fig. 2b). Using transcriptomics, one gene (Fig. 2c) was found to be differentially expressed. By mutant sequencing (Fig. 2d) two interesting variants were identified for gene 3 (presence of an indel, which may affect the functioning of the gene). Further investigations may be necessary for validation (screening for allelic variants in a wider germplasm pool, synteny analyses or functional analyses through gene editing and/or transformation, etc.). 


\section{Linking genetic maps with the physical sequence: demarcation of chromosomal regions}

Owing to the link between genetic maps and the physical genome sequences (see section 'Genome sequence and genomic tools in rose'), future studies may rapidly identify the sequence of flanking regions where genes of interest are located (Fig. 2a, b). Development of larger population sizes for the genetic studies will result in smaller target intervals, and hence fewer candidate genes. It is now also feasible to combine results from different genetic analyses, as demonstrated recently for the cloning of the major gene controlling the double-flower locus. For this, information from four different F1 progenies were combined to narrow down the sequence interval to a $293 \mathrm{~kb}$ region on chromosome $3^{5}$. A similar strategy could be employed for other major QTL using software such as BioMercator ${ }^{125}$. GWAS panels may also be combined if they share the set of SNP markers and if the phenotyping is done in the same way.

\section{From a sequence region to the best candidate genes}

The next step is to identify the best candidate gene(s) in the previously defined genomic region, using gene annotation and knowledge of the molecular basis of the studied trait (Fig. 2b). For example, a gene known to control flower development in Arabidopsis, an APETALA2 homologue, was identified in the genomic region of the DOUBLEFLOWER locus ${ }^{5}$. Similarly, an F-box protein and an SRNAse gene were identified in the region of the SI locus ${ }^{5}$.

Transcriptomic analyses (or meta-analysis using gene atlas) can be performed in parallel to identify differentially expressed genes, which also represent good candidates (Fig. 2c). For example, a genetic analysis identified a monoterpene synthesis locus on LG2 that co-located with a gene differentially expressed between scented and nonscented $\operatorname{roses}^{109}$. This led to the identification of RhNUDX1 as a likely candidate.

Direct sequencing of mutant pairs (sports, see also section 'Sports') is now an option to identify the functional mutation. This could involve targeted sequencing of the gene(s) located in genomic regions known to influence the trait (with techniques such as fluidigm or multiplex amplicon sequencing), sequencing of all the coding sequences (exome sequencing ${ }^{126}$, targeted sequencing with capture hybridization to subsets of genes $^{127}$ ) or even whole-genome re-sequencing ${ }^{128}$. This will potentially lead to the detection of important allelic variants, including non-synonymous mutations, insertions or deletions (Fig. 2d).

\section{Functional analysis}

A rapid and efficient genetic transformation protocol would greatly benefit functional gene studies in rose. The first publication on rose genetic transformation was in
$1994^{129}$. Since then, genes implied in colour, disease resistance, fragrance and architecture have been studied by stable genetic transformation in rose. In total only around 20 publications use this technique (Supplementary Table 1), probably due to the low overall efficiency $(\max 12 \%)$, the regeneration times required (6-12 months) and the fact that results appear to be highly cultivar-specific. Genetic information on loci and candidate genes involved in regeneration capacity were studied by Nguyen et al. $^{11,130}$ in 96 rose genotypes, offering some hope that regeneration protocols might be streamlined in future.

To circumvent such limitations in the meantime, some rose genes have been studied in heterologous systems, including Tobacco ${ }^{131}$, Pelargonium ${ }^{132}$ and Arabidopsis ${ }^{85,133-135}$. Systems for transient expression in rose have been developed by agroinfiltration in petals (e.g. refs. ${ }^{136,137}$ ) or leaves ${ }^{81}$, and by virus-induced gene silencing in leaves, axils, seedlings ${ }^{138}$ and petals ${ }^{139}$.

Using the rose genome and inferred protein sequence data, it will be easier to identify coding sequences of genes, alternative splicing proteins, promoter and regulatory element(s). These data could be used to define CRISPR-Cas9 guide RNAs, for example, using the website CRISPOR (http://crispor.tefor.net/) on which the Rose genome sequence is available. The improvement of genetic transformation efficiency would make the use of CRISPR-Cas9 technology feasible in rose.

\section{Rose breeding}

A strong demand for new cultivars still exists. This is reflected in the number of applications for plant breeders' right (PBR). In the European Union (EU), the Community Plant Variety Office (CPVO) administrates PBR. Since its inception in 1991, more than 61,000 applications were filed for ornamental plants, of which 4189 were for roses (all types) (CPVO 2018: https://cpvo.europa.eu/en/ applications-and-examinations/applications-and-titlesforce). More roses than those listed at CPVO have been commercialized in this period, as not all roses undergo PBR application (e.g. garden roses are rarely protected by PBR). However, it is indicative of a sustained output from the rose breeding sector.

\section{Potential marker deployment for traits in rose}

Many of the important morphological and agronomic traits in rose are controlled by single dominant loci, for example, miniature plant habit ${ }^{140}$, glossy foliage ${ }^{141}$, resistance to powdery mildew $\operatorname{Rpp}^{115}$ and black spot resistance and so on. As the phenotypes in a tetraploid progeny will strongly resemble one of the parents (Fig. 3), the dosage of the parents of the cross determines the fraction of offspring that will have the right phenotype, or be within the right range. To achieve a larger fraction of 


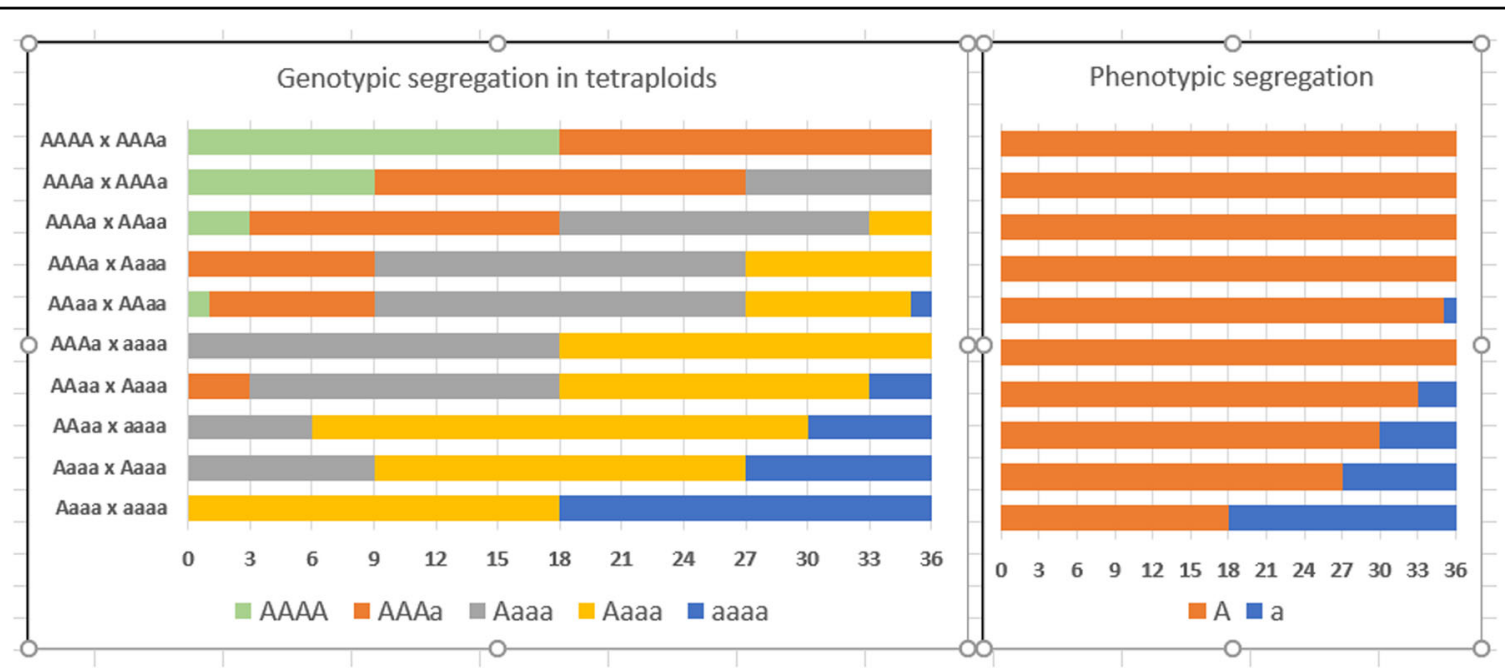

Fig. 3 Genotypic and phenotypic segregation ratios for a trait regulated by a single dominant gene with two alleles under random bivalent pairing in a tetraploid rose. At each locus, two alleles may occur in five different allelic states (nulliplex, simplex, duplex, triplex and quadruplex). The fraction of progeny of a cross with the desired phenotype will therefore differ significantly depending of the allelic state of the parents. Crosses of one parent carrying a dominant allele in simplex configuration to a homozygous recessive (nulliplex) second genotype will result in $50 \%$ of the progeny carrying the dominant allele (in simplex configuration). In autotetraploids, dominant traits are only guaranteed to be inherited in a cross when one of the parents has at least three copies of the dominant allele. Note that additivity, multiple alleles and the occurrence of double reduction have not been taken into account.

progeny carrying the trait, parents with higher allele dosages have to be combined (Fig. 3). For dominant traits allele dosage information cannot be obtained by phenotyping alone. Simplex can be distinguished from higher dosages by analysing a small progeny or an analysis of the pedigree. However, when two or more allele copies are present, the exact dosage cannot be inferred and needs be determined using linked SNP markers. Given the current strategies that breeders use for selection, the first steps in marker-assisted selection will most probably include selection of parents with optimized marker dosage for single genes of importance ${ }^{142}$.

Ornamental plant breeders often express concern that innovative traits may be missed by a marker-driven breeding programme. However, this assumes the breeding programme is entirely run by marker-assisted breeding without input from the 'breeder's eye'. This is unlikely to be the case ${ }^{19}$. Parental selection as outlined here only concerns optimizing the choice of the most optimal parent pairs out of many possible combinations of parents in view of an important trait in the offspring.

Determining the precise allelic configuration of QTLs in tetraploids is complicated, but advances in this direction have been made in recent years ${ }^{34,143}$. Markers whose parental phase is known (i.e. assigned to specific parental homologous chromosomes) could go a long way towards unravelling the allelic composition of important genes in the coming years. Transferring this knowledge into stable and selectable markers could be a very welcome development for the breeding community.
For quantitative traits, the marker-trait association may follow a dosage-dependent relationship if the allele dosage is additive ${ }^{5}$. For complex polygenic traits for which selection is not possible in the seedling stage, breeders could benefit from a better knowledge on genes that are in control, but a genomic selection approach (naive for the underlying genetics) might be useful as well. Examples of such traits are senescence (vase life), scent, disease resistance and plant architecture. One of the main criteria in cut rose selection is production, but this trait is hardly researched in roses from a genetic perspective.

\section{Multiple functional alleles at one locus}

Outbreeding tetraploids may contain multiple alleles at any single locus. A GWAS analysis of a set of tetraploid cultivars or accessions will almost certainly include multiple alleles at one locus, but even the two parents of a controlled cross may contain more than two different alleles among the eight alleles that segregate. Separate marker assays may therefore be needed to comprehensively tag all functional alleles, which may be challenging (or even impossible) at the single SNP level. One approach to circumvent this is to look for haplotypes in which allelic variants are either uniquely embedded or uniquely linked to a trait. However, reconstructing haplotypes from separate SNP assays for genic regions of interest is not straightforward in polyploids ${ }^{10}$. Obtaining haplotypes directly from sequencing reads is possible, but depends on SNP density, requires sufficient read depth, and is sensitive to sequencing errors ${ }^{22}$. Allele dosage 
inference from sequencing reads also is not straightforward, although methods and tools to deal with this are being developed ${ }^{24}$.

\section{Pyramiding $R$ genes}

Pyramiding of $R$ genes in a single plant genotype is important to increase the durability of disease resistance ${ }^{144,145}$. Pyramiding of $R$ genes is complicated by the fact that diagnostic pathotypes are required in phenotypic assays for the presence of a particular $R$ gene. Interactions between host genotypes and genotypes of the pathogen are often complex, involving multiple factors. Many pathotypes can be recognized by different $R$ genes, making them unsuitable as diagnostic pathotypes. As a consequence, DNA markers linked to specific R loci or those which are derived from the gene itself would be required to pyramid $R$ genes in roses and other crops. In roses, markers for $R d r 1$ have been derived from the gene directly ${ }^{146}$, whereas markers for $R d r 3$ and $R d r 4$ have been discovered by mapping these genes to particular regions of the rose genome ${ }^{33,114}$. Of particular interest are combinations of $R d r 1$ and $R d r 4$ as each of these genes confer broad-spectrum resistance to a range of black spot pathotypes. Furthermore, the genes have different genetic backgrounds and lie on different chromosomes. The region around Rdr4 does not contain sequences similar to $R d r 1$ (T.D. and M.L., unpublished results), which makes it less likely that the combined resistance specificity against most known isolates can be broken easily by pathogen adaptation.

Besides the difficulty of phenotypically distinguishing the effect of several resistance alleles, pyramiding resistances could create further problems. By combining different resistances from different breeding material (especially non-elite material, e.g. the introgression of $R d r 4$ from a climber rose to cut rose), many undesired alleles at other loci affecting other traits may be introduced. This linkage drag may necessitate multiple rounds of back-crossing and selection to recover resistant elite lines. Luckily, this process can be greatly accelerated using closely linked markers (coupled with a marker set which captures the elite genetic background). Owing to the genome sequence and the ease with which the donor and receptor plants can be re-sequenced, it should now be relatively straightforward to saturate regions surrounding $R$ genes with SNP markers, and enable marker-assisted back-crossing and gene pyramiding. In addition, GWAS using the information of ultra-dense SNP maps combined with the available genome sequences will enable the detection of minor resistance factors, which can be added to resistance pyramids.

\section{QTL analysis, association analysis and genomic selection}

QTL analysis studies often had a relatively low genetic resolution due to the limited population sizes used. In addition, only alleles present and segregating between the parents of the cross can be detected in such bi-parental studies. GWAS offer an alternative approach to identify genomic regions associated with specific traits in more diverse populations with potentially wider pools of interesting alleles. However, GWAS in rose panels that include multiple types of roses, or multiple species may suffer from serious confounding between population structure and allelic effects, leading to a loss of discriminatory power. Populations connected through common parents offer a compromise by enriching allelic diversity within the context of a balanced and controlled population structure ${ }^{56}$. They also reflect the types of populations generated during a breeding programme. Combining breeding and research in this way would represent an efficient use of resources.

Traits such as vase life, which cannot be scored in young plants, probably represent the first set of candidates for the deployment of molecular tools within a breeding programme. However, such traits are often complex or quantitative, for which molecular tools are less easily developed. A first step could be an analysis of the physiological components of vase life ending, as was done in chrysanthemum ${ }^{147}$. Depending on this, pertinent variables of vase life that may be more accurately phenotyped may be found. Subsequently, these determinants may be studied at the level of their underlying gene pathways. Given the complexity of such traits, genomic selection may prove to be a more appropriate approach. Genomic selection is an alternative method of increasing genetic gain in plant breeding programmes ${ }^{148}$. Whether it is suited to rose has yet to be demonstrated-as rose breeding involves the mixing of diverse genetic backgrounds that may distort the modelling of relationships in prediction models.

\section{Tailored breeding of rose}

Breeding goals in roses depend on their usage. In cut roses, production is a primary breeding goal and has yet to reach its full potential. Breeding companies differentiate their selection to specific production areas, resulting in the best-adapted cultivars for specific environments ${ }^{149}$. Disease resistance is needed to reduce the use of pesticides. Because of post-harvest transport of roses from areas of cultivation (e.g. in Africa and or South America) to consumers all over the world, diseases are not limited to the production environment, but also include postharvest diseases such as grey mould (Botrytis cinerea). Likewise, stress tolerance is important during cultivation, but also for vase life. Rose breeding therefore needs to incorporate many traits, ideally ones that are stably expressed across environments ${ }^{34,120}$.

Fragrance is gaining new interest because of consumer demand and the improved understanding of its 
underlying genetics. New traits in cut roses include novel flower shapes, colour evolution and glossiness of the foliage, and so on. In pot roses, plant habitus, flower colour, size and number of flowers per stem, number of petals, production and shelf life are the most important traits $^{149}$. In garden rose, disease resistance, fragrance, abundant, red and everblooming flowers were the traits of most interest among surveyed consumers ${ }^{150}$. Carefree roses are needed for landscaping and urban development - these are own-rooted roses supporting mechanical pruning, free of diseases and having an all-season decorative effect, for example, with colourful hips during wintertime. In the near future, because of climate change and expanding trade to more 'difficult' areas we may also need cultivars that have improved abiotic stress resistance, including frost tolerance and flowering during hot summers.

Clonally propagated plants can be easily replicated to establish a multi-environment set-up. Larger mapping populations, preferably replicated across multiple different environments, allow for genotype $\times$ environment interaction to be studied and understood. This may include RNA-sequencing eQTL mapping in GWAS $^{151}$.

Gene editing with CRISPR/Cas is interesting for rose as a novel tool for directed mutagenesis, as it can produce homozygous mutations in a polyploid ${ }^{152-154}$. Apart from restricted access by IP rights and constraints raised by legal issues in the $\mathrm{EU}^{155}$, the application of such new breeding techniques critically depends on the knowledge about gene functions. Although reference genome sequences are currently available, an approach using phenotypic and 'omics' data is needed in crops as the vast majority of genes remains uncharacterized ${ }^{156}$. Especially when a loss of function is aimed for, specific information on the targeted sequences is needed.

\section{Conclusions}

In the Rosaceous crops apple and peach, an integrated approach was carried out to fill the gap between available genome data and breeding in an EU-funded project: FruitBreedomics. Complementary approaches were used, including the development of tools and software, to help unravel the genetic control of the most important horticultural traits, as well as to develop plant material and methodologies for breeders. The project started through a consultation between breeders and researchers, aimed at cataloguing the most important traits. Overall, the project led to the development of new phenotyping tools to assess traits like fruit texture, and both biotic and abiotic stresses. Pre-breeding material with specific characterized traits was made available for breeders together with tools for the implementation of molecular markers in breeding ${ }^{157}$. Such a project may well help bridge the gap that still exists between rose research and breeding.
The slow adaptation of molecular techniques can, in some crops, be linked to the long breeding cycle (e.g. in fruit trees ${ }^{157}$ ), although poplar is an example of a tree with a long breeding cycle in which genomic studies were initiated many years ago ${ }^{158}$. For roses, breeding cycles are not particularly long, but a common platform for interaction between the different players (research and breeders) has been lacking. The recent rose genome sequencing projects that have been conducted were largely driven by research groups without support from the breeding industry. On the other hand, the WagRhSNP array, the high-density genetic maps, and GWAS studies have been developed with support of rose breeding companies.

The new tools (e.g. genome sequence, SNP array, software for dosage scoring and genetic mapping, etc.) and technology (e.g. next-generation sequencing) means that a new era has started for research in polyploid crops such as rose. Previous results on QTLs can be tied together, and new studies can build on this resource. Most resources are freely available at the GDR website ${ }^{13}$.

Genomic studies have opened up new routes to crop improvement, and this holds also for rose. We expect that researchers will use the resources to achieve faster progress in the fields described above. We hope that rose breeders will team up with researchers to discuss their needs and to start to incorporate marker-based selection methods to improve rose breeding for new demands within their breeding programmes.

\section{Acknowledgements}

We thank the editor, Zong-Ming (Max) Cheng, for initiating the Roadmap papers. M.J.M.S., P.A. and P.M.B. were supported in part by the TKI polyploids project 'Novel genetic and genomic tools for polyploid crops' (BO-26.03-009004 and BO-50-002-022).

\section{Author details}

${ }^{1}$ Plant Breeding, Wageningen University and Research, P.O. Box 386, 6700 AJ Wageningen, The Netherlands. ${ }^{2}$ Faculty of Natural Sciences, Institute for Plant Genetics, Molecular Plant Breeding, Leibniz University of Hannover,

Herrenhäuser Strasse 2, 30419 Hannover, Germany. ${ }^{3} \mid$ LVO, Plant Sciences Unit, Flanders Research Institute for Agriculture, Fisheries and Food, Caritasstraat 39,

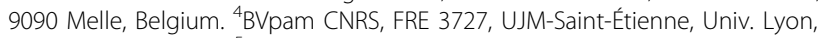
Saint-Etienne, France. ${ }^{5}$ IRHS, Agrocampus-Ouest, INRA, Université d'Angers, SFR 4207 QuaSaV, 42 rue Georges Morel BP 60057, 49071 Beaucouzé, France

\section{Authors' contributions}

M.J.M.S., P.M.B., P.A. and F.F. contributed to the tools section, M.J.M.S. and J.C. to genetic diversity, J.C. and T.R. to structural genomics, L.H.S.-O., T.D., M.L., S.B. and F.F. to functional genomics, P.A., T.D., M.L., J.d.R. and L.L. to breeding. P.M.B. produced Figs. 1 and 2, T.D. Fig. 3. M.J.M.S., P.A. and P.M.B. combined and streamlined the contributions. All authors edited the combined manuscript. M.J.M.S., P.A. and F.F. finalized the manuscript.

\section{Conflict of interest}

The authors declare that they have no conflict of interest.

Supplementary Information accompanies this paper at (https://doi.org/ 10.1038/s41438-019-0156-0) 
Received: 27 February 2019 Accepted: 18 April 2019

Published online: 03 May 2019

\section{References}

1. Barbier, F. et al. Sucrose is an early modulator of the key hormonal mechanisms controlling bud outgrowth in Rosa hybrida. J. Exp. Bot. 66, 2569-2582 (2015).

2. Spiller, M. et al. Towards a unified genetic map for diploid roses. Theor. Appl Genet. 122, 489-500 (2011).

3. Nakamura, N. Genome structure of Rosa multiflora, a wild ancestor of cultivated roses. DNA Res. 25, 113-121 (2018).

4. Raymond, O. et al. The Rosa genome provides new insights into the domestication of modern roses. Nat. Genet. 50, 772-777 (2018).

5. Hibrand Saint-Oyant, L. et al. A high-quality genome sequence of Rosa chinensis to elucidate ornamental traits. Nat. Plants 4, 473-484 (2018).

6. Roccia, A. et al. Biosynthesis of 2-phenylethanol in rose petals is linked to the expression of one allele of RhPAAS. Plant Physiol. https://doi.org/10.1104/ pp.18.01468 (2019).

7. Koning-Boucoiran, C. F. S. et al. Using RNA-Seq to assemble a rose transcriptome with more than 13,000 full-length expressed genes and to develop the WagRhSNP 68k Axiom SNP array for rose (Rosa L.). Front. Plant Sci. 6, 249 (2015)

8. Bourke, P. M. et al. Partial preferential chromosome pairing is genotype dependent in tetraploid rose. Plant J. 90, 330-343 (2017).

9. Vukosavljev, M. et al. High-density SNP-based genetic maps for the parents of an outcrossed and a selfed tetraploid garden rose cross, inferred from admixed progeny using the 68k rose SNP array. Hortic. Res. 3, 16052 (2016).

10. Bourke, P. M., Voorrips, R. E., Visser, R. G. F. \& Maliepaard, C. Tools for genetic studies in experimental populations of polyploids. Front. Plant Sci. 9, 513 (2018).

11. Nguyen, H. N., Schulz, D., Winkelmann, T. \& Debener, T. Genetic dissection of adventitious shoot regeneration in roses by employing genome-wide association studies. Plant Cell Rep. 36, 1493-1505 (2017).

12. Schulz, D. F. et al. Genome-wide association analysis of the anthocyanin and carotenoid contents of rose petals. Front. Plant Sci. 7, 1798 (2016).

13. Jung, S. et al. 15 years of GDR: new data and functionality in the Genome Database for Rosaceae. Nucleic Acids Res. 47, D1137-D1145 (2019).

14. Meng, J., Fougère-Danezan, M., Zhang, L.-B., Li, D.-Z. \& Yi, T.-S. Untangling the hybrid origin of the Chinese tea roses: evidence from DNA sequences of single-copy nuclear and chloroplast genes. Plant Syst. Evol. 297, 157-170 (2011).

15. Dubois, A. C. et al. Transcriptome database resource and gene expression atlas for the rose. BMC Genom. 13, 638 (2012).

16. Liu, X. et al. Comparative RNA-Seq analysis reveals a critical role for brassinosteroids in rose (Rosa hybrida) petal defense against Botrytis cinerea infection. BMC Genet. 19, 62 (2018).

17. Tian, X. et al. Genome-wide transcriptome analysis of the salt stress tolerance mechanism in Rosa chinensis. PLoS ONE 13, e0200938 (2018).

18. Neu, E. et al. Interaction of roses with a biotrophic and a hemibiotrophic leaf pathogen leads to differences in defense transcriptome activation. Plant Mol. Biol. https://doi.org/10.1007/s11103-018-00818-2 (2019).

19. Smulders, M.J.M., Arens, P. in Ornamental Crops, Handbook of Plant Breeding Vol. 11 (ed. Van Huylenbroeck, J.) 213-230 (Springer International Publishing AG, Cham, Switzerland, 2018).

20. Li, S. et al. Comparative transcriptomics identifies patterns of selection in roses. BMC Plant Biol. 18, 371 (2018).

21. Morin, P. A., Martien, K. K. \& Taylor, B. L. Assessing statistical power of SNPs for population structure and conservation studies. Mol. Ecol. Resour. 9, 66-73 (2009).

22. Motazedi, E., Finkers, R., Maliepaard, C. \& De Ridder, D. Exploiting nextgeneration sequencing to solve the haplotyping puzzle in polyploids: a simulation study. Brief Bioinform. 19, 387-403 (2018).

23. McKenna, A. et al. The Genome Analysis Toolkit: a MapReduce framework for analyzing next-generation DNA sequencing data. Genome Res. 20 1297-1303 (2010).

24. Gerard, D., Ferrão, L. F. V., Garcia, A. A. F. \& Stephens, M. Genotyping polyploids from Messy sequencing data. Genetics 210, 789-807 (2018).

25. Li, H. A statistical framework for SNP calling, mutation discovery, association mapping and population genetical parameter estimation from sequencing data. Bioinformatics 27, 2987-2993 (2011).
26. Davey, J. W. Genome-wide genetic marker discovery and genotyping using next-generation sequencing. Nat. Rev. Genet. 12, 499-510 (2011).

27. Yan, M. et al. Genotyping-by-sequencing application on diploid rose and a resulting high-density SNP-based consensus map. Hortic. Res. $\mathbf{5}$ 17 (2018).

28. Heo, M.-S. et al. Development of SNP markers using genotyping-bysequencing for cultivar identification in rose (Rosa hybrida). Hortic. Environ Biotechnol. 58, 292-302 (2017).

29. Therkildsen, N. O. \& Palumbi, S. R. Practical low-coverage genomewide sequencing of hundreds of individually barcoded samples for population and evolutionary genomics in nonmodel species. Mol. Ecol. Resour. 17, 194-208 (2017).

30. García-Lor, A., Luro, F., Navarro, L. \& Ollitrault, P. (2012) Comparative use of InDel and SSR markers in deciphering the interspecific structure of cultivated citrus genetic diversity: a perspective for genetic association studies. Mol. Genet. Genom. 287, 77-94 (2012).

31. Hackett, C. A., Boskamp, B. \& Vogogias, A. TetraploidSNPMap: Software for linkage analysis and QTL mapping in autotetraploid populations using SNP dosage data. J. Hered. 108, 438-442 (2017).

32. Bourke, P. M. PolymapR-linkage analysis and genetic map construction from F1 populations of outcrossing polyploids. Bioinformatics 34, 3496-3502 (2018).

33. Zurn, J. D. et al. Mapping a novel black spot resistance locus in the Climbing Rose Brite Eyes ${ }^{\mathrm{TM}}$ ('RADbrite'). Front. Plant Sci. 9, 1730 (2018).

34. Bourke, P. M. et al. Multi-environment QTL analysis of plant and flower morphological traits in tetraploid rose. Theor. Appl. Genet. 131, 2055-2069 (2018c).

35. Wylie, A. The history of garden roses. J. R. Hortic. Soc. 79, 555-571 (1954).

36. Maia, N. \& Venard, P. in Vénard, Cytotaxonomie du genre Rosa et origine des rosiers cultivés? Travaux sur le rosier de serre menés au C.R.A. d'Antibes. Fédération Nationale des Producteurs de l'Horticulture et des Pépinières, pp. 7-20 (1976).

37. Young, M. A., Schorr, P. \& Baer, R. Modern Roses 12 (American Rose Society, Shreveport, 2007).

38. Akond, M., Jin, S. \& Wang, X. Molecular characterization of selected wild species and miniature roses based on SSR markers. Sci. Hortic. 147, 89-97 (2012).

39. Tan, J. et al. Genetic relationships and evolution of old Chinese garden roses based on SSRs and chromosome diversity. Sci. Rep. 7, 15437 (2017).

40. Vukosavljev, M. et al. Genetic diversity and differentiation in roses: a garden rose perspective. Sci. Hortic. 162, 320-332 (2013).

41. Farooq, A. et al. Microsatellite analysis of Rosa damascena from Pakistan and Iran. Hortic. Environ. Biotechnol. 54, 141-147 (2013).

42. Liorzou, M. et al. Nineteenth century French rose (Rosa sp.) germplasm shows a shift over time from a European to an Asian genetic background. J. Exp. Bot. 67, 4711-4725 (2016).

43. Leus, L. et al. Molecular evaluation of a collection of rose species and cultivars by AFLP, ITS, rbc L, mat K. Acta Hortic. 651, 141-147 (2004).

44. Koopman, W. J. M. et al. AFLP markers as a tool to reconstruct complex relationships: a case study in Rosa (Rosaceae). Am. J. Bot. 95 353-366 (2008)

45. Fernández-Romero, M.D. et al. in Cytological and Molecular Characterisation of a Collection of wild and cultivated roses. Roses. Vol. 3, (ed. Zlesak, D. C.) 28-39 (Floriculture and Ornamental Biotechnology Global Science Books, London, 2009).

46. Qiu, X. Q. et al. Genetic relationships of wild roses, old garden roses, and modern roses based on internal transcribed spacers and matK sequences. Hortscience 48, 1445-1451 (2013).

47. Valentina, S., Aziz, A. \& Roberto, B. Characterization and genetic relationships of wild species and old garden roses based on microsatellite analysis. J. Am. Soc. Hortic. Sci. 131, 66-73 (2006).

48. Vukosavljev, M. et al. Genetic diversity and differentiation in roses: A garden rose perspective. Sci. Hortic. (Amsterdam) 162, 320-332 (2013).

49. Gardes, L., Heizmann, P. \& Joyaux, F. Molecular typing and history of the provins roses horticultural group. Eur. J. Hortic. Sci. 70, 162-172 (2005).

50. $\mathrm{Wu}, \mathrm{G}$. A. et al. Sequencing of diverse mandarin, pummelo and orange genomes reveals complex history of admixture during citrus domestication. Nat. Biotechnol. 32, 656 (2014).

51. Cornille, A. et al. New Insight into the history of domesticated apple: secondary contribution of the European wild apple to the genome of cultivated varieties. PLOS Genet. 8, e1002703 (2012). 
52. Besnard, G. et al. Population genetics of Mediterranean and Saharan olives: geographic patterns of differentiation and evidence for early generations of admixture. Ann. Bot. 112, 1293-1302 (2013).

53. Rosyara, U. R. et al. Fruit size QTL identification and the prediction of parental QTL genotypes and breeding values in multiple pedigreed populations of sweet cherry. Mol. Breed. 32, 875-887 (2013)

54. Van de Weg, W. E. et al. Pedigree genotyping: a new pedigree-based approach of QTL identification and allele mining by exploiting breeding material. Acta Hortic. 708, 483-488 (2006).

55. Fresnedo-Ramírez, J., Frett, T. J. \& Sandefur, P. J. et al. QTL mapping and breeding value estimation through pedigree-based analysis of fruit size and weight in four diverse peach breeding programs. Tree Genet. Genom. 12, 25 (2016)

56. Howard, N. P. et al. Two QTL characterized for soft scald and soggy breakdown in apple (Malus $\times$ domestica) through pedigree-based analysis of a large population of interconnected families. Tree Genet. Genom. 14, 2 (2018).

57. Kouassi, A. B. et al. Estimation of genetic parameters and prediction of breeding values for apple (Malus $\times$ domestica) fruit quality traits using pedigreed plant material in. Eur. Tree Genet. Genom. 5, 659-672 (2009).

58. Bink, M. C. A. M. et al. Bayesian QTL analyses using pedigreed families of an outcrossing species, with application to fruit firmness in apple. Theor. Appl. Genet. 127, 1073-1090 (2014)

59. Mangandi, J. et al. Pedigree-based analysis in a multiparental population of octoploid strawberry reveals QTL alleles conferring resistance to Phytophthora cactorum. G3 7, 1707-1719 (2017).

60. Proïa, F., Panloup, F., Trabelsi, C. \& Clotault, J. Probabilistic reconstruction of genealogies for polyploid plant species. J. Theor. Biol. 462, 537-551 (2019).

61. Smulders, M. J. M., Esselink, D., Voorrips, R. E. \& Vosman, B. Analysis of a database of DNA profiles of 734 hybrid tea rose varieties. Acta Hortic. $\mathbf{8 3 6}$ 169-175 (2009).

62. Blackburn, K. B. \& Harrison, J. H. The status of the British rose forms as determined by their cytological behaviour. Ann. Bot. 35, 159-188 (1921).

63. Täckholm, G. On the cytology of the genus Rosa. Sven. Bot. Tidskr. 14 300-311 (1920).

64. Zlesak, D. C. Pollen diameter and guard cell length as predictors of ploidy in diverse rose cultivars, species, and breeding lines. Floric. Ornam. Biotechnol. $\mathbf{3}$, 53-70 (2009).

65. Nybom, H., Esselink, G. D., Werlemark, G. \& Vosman, B. Microsatellite DNA marker inheritance indicates preferential pairing between two highly homologous genomes in polyploid and hemisexual dog-roses, Rosa L. Sect. Canina DC. Heredity. 92, 139 (2003).

66. Lim, K. Y. et al. Evolutionary implications of permanent odd polyploidy in the stable sexual, pentaploid of Rosa canina L. Heredity 94, 501-506 (2005).

67. Yokoya, K., Roberts, A. V., Mottley, J., Lewis, R. \& Brandham, P. E. Nuclear DNA Amounts In Roses. Ann. Bot. 85, 557-561 (2000).

68. Jian, $\mathrm{H}$. et al. Nuclear DNA content and $1 \mathrm{Cx}$-value variations in genus Rosa $\mathrm{L}$. Caryologia 67, 273-280 (2014).

69. Bennetzen, J. L., Ma, J. \& Devos, K. M. Mechanisms of recent genome size variation in flowering plants. Ann. Bot. 95, 127-132 (2005).

70. Leitch, A. R. \& Leitch, I. J. Genomic plasticity and the diversity of polyploid plants. Science 320, 481-483 (2008).

71. Iwata, $\mathrm{H}$. et al. The TFL1 homologue $\mathrm{KSN}$ is a regulator of continuous flowering in rose and strawberry. Plant J. 69, 116-125 (2012).

72. Klein, S. J. \& O'Neill, R. J. Transposable elements: genome innovation, chromosome diversity, and centromere conflict. Chromosom. Res. 26, 5 (2018).

73. Shulaev, V. et al. The genome of woodland strawberry (Fragaria vesca). Nat. Genet. 43, 109-116 (2011).

74. Zhang, J. et al. The diploid origins of allopolyploid rose species studied using single nucleotide polymorphism haplotypes flanking a microsatellite repeat. J. Hortic. Sci. Biotechnol. 88, 85-92 (2013).

75. Cao, J. et al. Whole-genome sequencing of multiple Arabidopsis thaliana populations. Nat. Genet. 43, 956-963 (2011).

76. Yao, W., Li, G., Zhao, H., Wang, G., Lian, X. \& Xie, W. Exploring the rice dispensable genome using a metagenome-like assembly strategy. Genome Biol. 16, 187 (2015)

77. Hirsch, C. N., Foerster, J. M. \& Johnson, J. M. et al. Insights into the maize pangenome and pan-transcriptome. Plant Cell 26, 121-135 (2014).

78. Wang, W. et al. Genomic variation in 3,010 diverse accessions of Asian cultivated rice. Nature 557, 43-49 (2018).
79. Hardigan, M. A. et al. Genome reduction uncovers a large dispensable genome and adaptive role for copy number variation in asexually propagated Solanum tuberosum. Plant Cell 28, 388 (2016).

80. Wang, W. et al. High rate of chimeric gene origination by retroposition in plant genomes. Plant Cell 18, 1791 (2006).

81. Terefe-Ayana, D. et al. Mining disease-resistance genes in roses: functional and molecular characterization of the Rdr1 locus. Front. Plant Sci. 2, 35 (2011)

82. Terefe-Ayana, D., Kaufmann, H., Linde, M. \& Debener, T. Evolution of the Rdr1 TNL-cluster in roses and other Rosaceous species. BMC Genom. 13, 409 (2012).

83. Debener, T. \& Linde, M. Exploring complex ornamental genomes: the rose as a model plant. Crit. Rev. Plant Sci. 28, 267-280 (2009).

84. Randoux, M. et al. Gibberellins regulate the transcription of the continuous flowering regulator, RoKSN, a rose TFL1 homologue. J. Exp. Bot. 63 6543-6554 (2012).

85. Randoux, M. et al. RoKSN, a floral repressor, forms protein complexes with RoFD and RoFT to regulate vegetative and reproductive development in rose. N. Phytol. 202, 161-173 (2014).

86. Wang, Z . et al. The divergence of flowering time modulated by FT/TFL1 is independent to their interaction and binding activities. Front. Plant Sci. 8, 697 (2017).

87. Roman, $\mathrm{H}$. et al. Genetic analysis of the flowering date and number of petals in rose. Tree Genet. Genom. 11, 1-13 (2015).

88. Dubois, A et al. Tinkering with the C-function: a molecular frame for the selection of double flowers in cultivated roses. PLOS ONE 5, e9288 (2010).

89. Debener, T. \& Mattiesch, L. Construction of a genetic linkage map for roses using RAPD and AFLP markers. Theor. Appl. Genet. 99, 891-899 (1999).

90. Han, Y. et al. An APETALA2 Homolog, RcAP2, regulates the number of rose petals derived from stamens and response to temperature fluctuations. Front. Plant Sci 9, 481 (2018).

91. François, L. et al. A miR172 target-deficient AP2-like gene correlates with the double flower phenotype in roses. Sci. Rep. 8, 12912 (2018).

92. Gattolin, S. et al. Deletion of the miR172 target site in a TOE-type gene is a strong candidate variant for dominant double-flower trait in Rosaceae. Plant J. 96, 358-371 (2018)

93. Han, Y. et al. Comparative RNA-seq analysis of transcriptome dynamics during petal development in Rosa chinensis. Sci. Rep. 7, 43382 (2017).

94. Jay, M. et al. in Encyclopedia of Rose Science (eds Gudin, S. \& Debener, T.) 248-255 (Elsevier, Oxford, 2003)

95. Ogata, J., Kanno, Y., Itoh, Y., Tsugawa, H. \& Suzuki, M. Anthocyanin biosynthesis in roses. Nature 435, 757-758 (2005).

96. Holton, T. A. \& Tanaka, Y. Blue roses-a pigment of our imagination? Trends Biotechnol. 12, 40-42 (1994).

97. Gitonga, V. W. et al. Inheritance and OTL analysis of the determinants of flower color in tetraploid cut roses. Mol. Breed. 36, 143 (2016).

98. Ueda, Y. Takeshita, D. \& Ando, T. Pollination in Rosa rugosa Thunb. ex Murray. Acta Hortic. 424, 309-310 (1996).

99. Sassa, H. Molecular mechanism of the S-RNase-based gametophytic selfincompatibility in fruit trees of Rosaceae. Breed. Sci. 66, 116-121 (2016).

100. Debener, T. et al. Genetic and molecular analysis of key loci involved in self incompatibility and floral scent in roses. Acta Hortic. 870, 183-190 (2010).

101. Bergougnoux, $\mathbf{V}$. et al. Both the adaxial and abaxial epidermal layers of the rose petal emit volatile scent compounds. Planta 226, 853-866 (2007).

102. Scalliet, G. et al. Scent evolution in Chinese roses. Proc. Natl Acad. Sci. USA 105, 5927-5932 (2008)

103. Guterman, I. et al. Rose Scent. Genomics approach to discovering novel flora fragrance-related genes. Plant Cell 14, 2325-2338 (2002).

104. Kaminaga, Y. et al. Plant phenylacetaldehyde synthase is a bifunctional homotetrameric enzyme that catalyzes phenylalanine decarboxylation and oxidation. J. Biol. Chem. 281, 23357-23366 (2006).

105. Chen, X.-M. et al. Functional characterization of rose phenylacetaldehyde reductase (PAR), an enzyme involved in the biosynthesis of the scent compound 2-phenylethanol. J. Plant Physiol. 168, 88-95 (2011).

106. Hirata, $\mathrm{H}$. et al. Functional characterization of aromatic amino acid aminotransferase involved in 2-phenylethanol biosynthesis in isolated rose petal protoplasts. J. Plant Physiol. 169, 444-451 (2012).

107. Hirata, $\mathrm{H}$. et al. Seasonal induction of alternative principal pathway for rose flower scent. Sci. Rep. 6, 20234 (2016).

108. Tholl, D. in Biotechnology of Isoprenoids (eds Schrader, J. \& Bohlmann, J.) 63-106 (Springer International Publishing, Cham, 2015). 
109. Magnard, J. L. et al. Biosynthesis of monoterpene scent compounds in roses. Science 349, 81-83 (2015)

110. Cherri-Martin, M., Jullien, F., Heizmann, P. \& Baudino, S. Fragrance heritability in hybrid Tea roses. Sci. Hortic. 113, 177-181 (2007).

111. Ma, N. et al. Petal senescence: a hormone view. J. Exp. Bot. 69, 719-732 (2018).

112. Debener, T. \& Byrne, D. H. Disease resistance breeding in rose: current status and potential of biotechnological tools. Plant Sci. 228, 107-117 (2014).

113. Von Malek, B. \& Debener, T. Genetic analysis of resistance to blackspot (Diplocarpon rosae) in tetraploid roses. Theor. Appl. Genet. 96, 228-231 (1998).

114. Whitaker, V. W. et al. Rdr3, a novel locus conferring black spot disease resistance in tetraploid rose: genetic analysis, LRR profiling, and SCAR marker development. Theor. Appl. Genet. 120, 573-585 (2010).

115. Linde, M. \& Debener, T. solation and identification of eight races of powdery mildew of roses (Podosphaera pannosa) (Wallr:Fr) de Bary and the genetic analysis of the resistance gene Rpp 1. Theor. Appl. Genet. 107, 256-262 (2003).

116. Linde, M., Hattendorf, A., Kaufmann, H. \& Debener, T. Powdery mildew resistance in roses: QTL mapping in different environments using selective genotyping. Theor. Appl. Genet. 113, 1081-1092 (2006).

117. Hosseini Moghaddam, $\mathrm{H}$. et al. Construction of a genetic linkage map with SSR, AFLP and morphological markers to locate QTLs controlling pathotypespecific powdery mildew resistance in diploid roses. Euphytica 184, 413-427 (2012)

118. Jupe, F. et al. Resistance gene enrichment sequencing (RenSeq) enables reannotation of the NB-LRR gene family from sequenced plant genomes and rapid mapping of resistance loci in segregating populations. Plant J. 76, 530-544 (2013)

119. Armstrong, M. R. et al. Tracking disease resistance deployment in potato breeding by enrichment sequencing. Plant Biotechnol. J. 17, 540-549 (2019).

120. Gitonga, V. W. et al. Genetic variation, heritability and genotype by environment interaction of morphological traits in a tetraploid rose population. BMC Genet. 15, 1 (2014).

121. Kawamura, $\mathrm{K}$. et al. Inheritance of garden rose architecture and its association with flowering behaviour. Tree Genet. Genom. 11, 22 (2015).

122. Kawamura, K. et al. Quantitative trait loci for flowering time and inflorescence architecture in rose. Theor. Appl. Genet. 122, 661-675 (2011).

123. Li-Marchetti, C. et al. 3D phenotyping and QTL analysis of a complex character: rose bush architecture. Tree Genet. Genom. 13, 112 (2017).

124. Biber, A. et al. Molecular markers from a BAC contig spanning the Rdr1 locus: a tool for marker-assisted selection in roses. Theor. Appl. Genet. 120, 765-773 (2010).

125. Sosnowski, O., Charcosset, A. \& Joets, J. BioMercator V3: an upgrade of genetic map compilation and quantitative trait loci meta-analysis algorithms. Bioinformatics 28, 2082-2083 (2012).

126. Krasileva, K. V. et al. Uncovering hidden variation in polyploid wheat. Proc. Natl Acad. Sci. USA 114, E913-E921 (2017).

127. Steuernagel, B. et al. Rapid cloning of disease-resistance genes in plants using mutagenesis and sequence capture. Nat. Biotechnol. 34, 652-655 (2016).

128. Li, W.-T. et al. Whole-genome resequencing reveals candidate mutations for pig prolificacy. Proc. R. Soc. B 284, 20172437 (2017).

129. Firoozabady, E., Moy, Y., Courtney-Gutterson, N. \& Robinson, K. Regeneration of transgenic rose (Rosa hybrida) plants from embryogenic tissue. Nat. Biotechnol. 12, 609-613 (1994).

130. Nguyen, T. H. N., Winkelmann, T. \& Debener, T. Development of markers for shoot organogenesis in roses. Acta Hortic. 1232, 7-14 (2019).

131. Xing, S. et al. Expression vector construction and genetic transformation of Rosa rugosa beta-1,3-glucanase gene (RrGlu). Am. J. Plant Sci. 8, 7 (2017).

132. Hamama, L. et al. Overexpression of RoDELLA impacts the height, branching, and flowering behaviour of Pelargonium $\times$ domesticum transgenic plants. Plant Cell Rep. 31, 2015-2029 (2012).

133. Xu, K. D. et al. A MADS-box gene associated with protocorm-like body formation in Rosa canina alters floral organ development in Arabidopsis. Can. J. Plant Sci. 98, 309-317 (2018).

134. Jiang, G. M. et al. The Rose (Rosa hybrida) NAC transcription factor 3 gene RhNAC3, involved in ABA signaling pathway both in Rose and Arabidopsis. PLOS ONE 9, https://doi.org/10.1371/journal.pone.0109415 (2014).
135. Jiang, X. Q. et al. The novel rose MYB transcription factor RhMYB96 enhances salt tolerance in transgenic Arabidopsis. Plant Mol. Biol. Rep. 36, 406-417 (2018).

136. Yasmin, A. \& Debener, T. Transient gene expression in rose petals via Agrobacterium infiltration. Plant Cell Tiss. Org. Cult. 102, 245-250 (2010).

137. Zeinipour, M. et al. Agroinfiltration: a rapid and reliable method to select suitable rose cultivars for blue flower production. Physiol. Mol. Biol. Plants 24, 503-511 (2018).

138. Tian, J. et al. TRVGFP: a modified Tobacco rattle virus vector for efficient and visualizable analysis of gene function. J. Exp. Bot. 65, 311-322 (2014).

139. Dai, F. W. et al. RhNAC2 and RhEXPA4 are involved in the regulation of dehydration tolerance during the expansion of rose petals. Plant Physiol. 160 2064-2082 (2012).

140. Dubois, L. A. M. \& De Vries, D. P. On the inheritance of the dwarf character in Polyantha $\times$ Rosa chinensis minima (Sims) Voss F1-populations. Euphytica 36, 535-539 (1987).

141. Lammerts, W. E. The Scientific Basis of Rose Breeding. American Rose Annual (1945). http://bulbnrose.x10.mx/Roses/breeding/Lammerts/Lammerts_yellow. html.

142. Smulders, M. J. M. et al. Some thoughts on how to use markers in tetraploid rose breeding. Acta Hortic. 1232, 1-9 (2019).

143. Hackett, C. A., Bradshaw, J. E. \& Bryan, G. J. QTL mapping in autotetraploids using SNP dosage information. Theor. Appl. Genet. 127, 1885-1904 (2014).

144. Mundt, C. C. Pyramiding for resistance durability: theory and practice. Phytopathology 108, 792-802 (2018).

145. Koller, T, Brunner, S, Herren, G. Hurn, S. \& Keller, B. Pyramiding of transgenic $\mathrm{Pm} 3$ alleles in wheat results in improved powdery mildew resistance in the field. Theor. Appl. Genet. 131, 861-871 (2018).

146. Menz, I., Straube, J., Linde, M. \& Debener, T. The TNL gene Rdr1 confers broad-spectrum resistance to Diplocarpon rosae. Mol. Plant Pathol. 19 1104-1113 (2018).

147. Van Geest, G. et al. Breeding for postharvest performance in chrysanthemum by selection against storage-induced degreening of disk florets. Postharvest Biol. Technol. 124, 45-53 (2017).

148. Bernardo, R. Bandwagons I, too, have known. Theor. Appl. Genet. 129 2323-2332 (2016).

149. Leus, L., Van Laere, L., De Riek, J. Van Huylenbroeck, J. in Ornamental Crops, Handbook of Plant Breeding Vol. 11 (ed. Van Huylenbroeck, J.) 719-767 (Springer International Publishing AG, Cham, Switzerland, 2018).

150. Waliczek, T. M., Byrne, D. H. \& Holeman, D. J. Growers' and consumers' knowledge, attitudes and opinions regarding roses available for purchase Acta Hortic. 1064, 235-239 (2015).

151. Ingvarsson, P. K. \& Street, N. R. Association genetics of complex traits in plants. N. Phytol. 189, 909-922 (2011).

152. Nadakuduti, S. S. et al. Genome editing for crop improvement-applications in clonally propagated polyploids with a focus on potato (Solanum tuberosum L.). Front. Plant Sci. 9, 1607 (2018).

153. Schaart, J. G., Van de Wiel, C. C. M., Lotz, L. A. P. \& Smulders, M. J. M Opportunities for products of new plant breeding techniques. Trends Plant Sci. 21, 438-449 (2016)

154. Cheng, J. et al. Detection of induced mutations in CaFAD2 genes by nextgeneration sequencing leading to the production of improved oil composition in Crambe abyssinica. Plant Biotechnol. J. 13, 471-481 (2015).

155. Jouanin, A., Boyd, L. A., Visser, R. G. F. \& Smulders, M. J. M. Development of wheat with hypoimmunogenic gluten obstructed by the gene editing policy in Europe. Front. Plant Sci. 9, 1523 (2018).

156. Scheben, A. \& Edwards, D. Bottlenecks for genome-edited crops on the road from lab to farm. Genome Biol. 19, 178 (2018).

157. Laurens, F. et al. An integrated approach for increasing breeding efficiency in apple and peach in Europe. Hortic. Res. 5, 11 (2018).

158. Yang, $X$. et al. Poplar genomics: state of the science. Crit. Rev. Plant Sci. $\mathbf{2 8}$ 285-308 (2009).

159. Delcher, A. L., Salzberg, S. L. \& Phillippy, A. M. Using MUMmer to identify similar regions in large sequence sets. Curr. Protoc. Bioinform. 10, Unit 10.3 (2003). 